\title{
Palladium catalysed asymmetric alkylation of benzophenone Schiff base glycine esters in ionic liquids
}

\author{
DAE HYUN KIM ${ }^{\mathrm{a}}$, JIN KYU IM ${ }^{\mathrm{a}}$, DAE WON KIM ${ }^{\mathrm{a}}$, MINSERK CHEONG ${ }^{\mathrm{a}}$, \\ HOON SIK KIM $^{\mathrm{a}, *}$ and DEB KUMAR MUKHERJEE ${ }^{\mathrm{b}, *}$ \\ a Department of Chemistry and Research institute for Basic Sciences, Kyung Hee University, 1 Hoegi-Dong, \\ Dongdaemun-Gu, Seoul 130-701, Republic of Korea \\ ${ }^{\mathrm{b}}$ Department of Chemistry, Ramsaday College, Amta, Howrah 711401, India \\ e-mail: debkumarmukherjee@rediffmail.com; khs2004@khu.ac.kr
}

MS received 1 January 2011; revised 4 March 2011; accepted 3 May 2011

\begin{abstract}
Asymmetric alkyl substitution of various benzophenone Schiff base substrates under biphasic conditions proceeded using optically active Palladium(II) complexes. The corresponding products were obtained in high yields but with moderate enantiomeric excess (ee). Addition of specific ionic liquids to the reaction medium enhanced reactivity and selectivity for phase transfer catalytic (PTC) glycine alkylation. It has been found that there is an anionic influence of the ionic liquids that modify the steric environment around the enolate ion. A computer-assisted molecular design of enantioselective phase-transfer catalysis with the palladium complex and the ionic liquid has been done.
\end{abstract}

Keywords. Asymmetric alkylation; Schiff base; phase-transfer catalysis; chiral ionic liquid.

\section{Introduction}

Asymmetric phase transfer catalysis offers a powerful method for many reactions including the synthesis of unnatural amino acids. ${ }^{1-4}$ The first general synthesis of racemic amino acids by phase transfer catalysis (PTC) based on the alkylation of glycine ester Schiff bases was reported in $1978 .{ }^{5}$ In 1989 chiral catalysts derived from the cinchona alkaloids afforded optically active amino acids in a room temperature enantioselective PTC process. ${ }^{6}$ A second generation of chiral quaternary ammonium salts, the N,O- dialkylated cinchona salts, gave impressive enantioselectivities (79$80 \%$ ee). ${ }^{7,8}$ A third generation of chiral PTC catalysts has also been reported independently by Corey and Lygo groups. ${ }^{9,10}$ In these reactions a variety of alkyl halides were used and either enantiomer of the product could be prepared by selective use of the pseudoenantiomeric cinchonidine ${ }^{11,12}$ or cinchonine ${ }^{13,14}$ derived catalysts. Further modifications of the catalytic system include dimeric, ${ }^{15}$ trimeric $^{16}$ and other stereogenic centers ${ }^{17,18}$ which allow many options for improving greater reactivity and selectivity. Polymer supported

\footnotetext{
${ }^{*}$ For correspondence
}

quaternary ammonium salts have also been successfully used in the asymmetric alkylation of a glycine derivative by various groups. ${ }^{19-25}$ Recovered polymeric catalysts show high selectivity and can be used again for the same reaction.

Although there are successful catalysts for phase transfer alkylation reaction, formation of $\mathrm{C}-\mathrm{C}$ bonds through a direct alkylation using orthopalladated complex was unknown and has not been reported so far. Only one group, ${ }^{26}$ as far as our knowledge goes, has earlier used chiral copper and cobalt salen complexes as phase-transfer catalysts for asymmetric synthesis of $\alpha$-methyl $\alpha$-amino acids. We have been interested for some time in the application of phase transfer catalysis to the preparation of amino acid derivatives. The optically active orthopalladated phenanthrylamine phase transfer catalyst has been produced and explored for asymmetric glycine alkylation by us. ${ }^{27}$ The sterically hindered orthopalladated complex provided asymmetric induction in benzophenone Schiff base substrates under biphasic conditions. Prompted by the successful results from the phenanthrylamine moiety in PTC, we focused on different orthopalladated complexes hypothesizing that with the proper alignment of the ammonium centers, optimum enantioselectivity would be observed. We have reported ${ }^{28}$ a set of chiral palladium complexes which were effective in asymmetric induction of a range 
of electrophiles. The ee content was however moderate in each case and effort was on to increase it.

Screening of different reaction conditions show that low temperature $\left(-20^{\circ} \mathrm{C}\right.$ to $\left.0^{\circ} \mathrm{C}\right)$ and dichloromethane or a mixture of toluene:chloroform $(7 / 3, \mathrm{~V} / \mathrm{V})$ as solvents are effective in promoting the displacement reactions under phase transfer conditions. Recently the environmentally benign room temperature ionic liquids emerged as an alternative reaction media for chemical processes including chemical catalysis. ${ }^{29-31}$ Several groups have recently demonstrated ${ }^{32,33}$ the use of room temperature ionic liquid as a catalyst and solvent for the cyanide displacement of benzyl chloride and alkylation of benzyl cyanide under phase transfer conditions. Some have even reported simple transformation of chiral natural anions to liquid medium and their use to induce chirality. ${ }^{34-36}$ This prompted us to explore the alkylation of $\mathrm{N}$-diphenylmethylene glycine esters in chiral ionic liquid medium using optically active palladium(II) complexes. We report here the combination of an asymmetric palladium catalyst and a prochiral ionic solvent that promotes glycine alkylation under mild conditions. The strategy adopted could broaden both understanding and applicability.

$$
\text { (Alkylated enantiomer) }
$$

\section{Experimental}

Analytical grade reagents and freshly distilled solvents were used throughout our investigation. The palladium complexes were prepared and characterized as per standard literature methods with slight modifications done to suit our experimental conditions. The synthetic procedure and analytical data of the compounds can be found in a previous communication by the authors. 1-Butyl-3-methylimidazolium based ionic liquids like $[\mathrm{BMIm}] \mathrm{MeHPO}_{3},[\mathrm{BMIm}] \mathrm{MeSO}_{4},[\mathrm{BMIm}] \mathrm{CH}_{3} \mathrm{COO}$ and $[\mathrm{BMIm}] \mathrm{Tf}_{2} \mathrm{~N}$ were prepared by known literature methods ${ }^{37,38}$ and the ${ }^{1} \mathrm{H}$ NMR spectra of the assynthesized liquids are provided in the supporting documents accompanying the manuscript. The ionic liquid ( $N, N$-dimethylephedriniumbis-(trifluoromethanesulfon) imidate) has been reported ${ }^{39}$ to be a solid $\left(\mathrm{mp}=54^{\circ} \mathrm{C}\right)$ but we have actually isolated the liquid at a vacuum of $0.04 \mathrm{mbar}$ and used it in the reaction medium. All these ionic liquids were vacuum dried at $70^{\circ} \mathrm{C}$ prior to use to free them from moisture contamination. Ionic liquids like $[\mathrm{BMIm}] \mathrm{Cl},[\mathrm{BMIm}] \mathrm{BF}_{4}$ and $[\mathrm{BMIm}] \mathrm{PF}_{6}$ were however obtained from Aldrich chemicals and used as received.

The Schiff base Ethyl-N-diphenylmethyleneglycinate (substrate I) was found to be unstable over the silica gel slides and always produced two visible spots (visualized under UV light). The spot at $\mathrm{R}_{\mathrm{f}}=0.42$ has been assigned to the imine and the other spot at $\mathrm{R}_{\mathrm{f}}=$ 0.70 corresponds to Benzophenone (eluent, $n$-Hexane/
EtOAc, 9:1). The Schiff base was found to be unstable even in neutral alumina plates. Astonishingly, none of the recent literature has cited this until we came across an article published by Martin J O'Donnel and coworkers. ${ }^{40} \quad$ Tert-butyl-N-diphenylmethyleneglycinate (substarte II) was also used for alkylation with methyl iodide and benzyl bromide as alkylating agents. The Schiff base derivatives of glycine were chromatographed on grade $\mathrm{V}$ basic alumina with ethyl acetate in hexane as eluant. Vibrational, electronic and ${ }^{1} \mathrm{H}$ NMR spectra were taken with Perkin Elmer 883, Shamadzu MPC-3700 and Bruker $400 \mathrm{MHz}$ instruments, respectively. The enantioselectivity was determined by chiral HPLC analysis (chiracel OD, hexane: 2-propanol (99.5:0.5), flow rate $1 \mathrm{ml} / \mathrm{min}, 20^{\circ} \mathrm{C}, \lambda=$ $254 \mathrm{~nm}$ ). Optical rotation studies wherever necessary were determined on a Perkin Elmer 241 polarimeter using $1 \%$ solution in $\mathrm{CH}_{2} \mathrm{Cl}_{2}$.

\subsection{Experimental procedure for the Pd-catalysed asymmetric alkylation}

Palladium complex $(0.05 \mathrm{mmol})$ was dissolved in methylene chloride $(1 \mathrm{~mL})$. Aqueous $\mathrm{KOH}$ solution $(50 \%, 0.5 \mathrm{~mL})$ was added to a vigorously stirred solution of the Schiff base $(0.2 \mathrm{~g}, 0.70 \mathrm{mmol})$, metal complex and benzyl bromide (1.2 eq, $0.1 \mathrm{~mL}, 0.85 \mathrm{mmol}$ ) in methylene chloride. Chiral ionic liquid $(0.5 \mathrm{ml})$ was added to the mixture at room temperature almost 
immediately and stirred for several hours. The course of the reaction was followed by TLC (eluent, $n$-hexane/ethylacetate, 90/10) until the starting material has been consumed. The suspension was diluted with diethylether and the combined organic extracts were dried over $\mathrm{Na}_{2} \mathrm{SO}_{4}$, filtered and concentrated in vacuum. Purification of the residue by flash column chromatography on alumina 90 active basic (0.063$0.200 \mathrm{~mm}$ ) afforded the alkylated product as colourless oil (yield generally $>80 \%$ ).

\section{Results and discussion}

\subsection{Asymmetric alkylation of benzophenone imine glycinate under phase transfer conditions using optically active palladium complexes}

The orthopalladated dimeric complexes (Catalyst-I-III) and the dimeric R-complex of the benzylamine variety (catalyst-IV) were prepared by known literature methods and these were then used as catalyst precursors for phase transfer alkylation reactions. Though acetato-, chloro- and bromo-bridged orthopalladated complexes were prepared and reported in a previous article by us, ${ }^{28}$ we have used only the chloro-bridged chiral palladium complexes in the present context. 50\% Aqueous potassium hydroxide solution and 1.2 equivalents of the alkylating agent was used in most of the runs under biphasic conditions. All Asymmetric alkylation reactions were conducted at room temperature $\left(20^{\circ} \mathrm{C}\right)$.

To examine the effect of the chiral as well as achiral additives in liquid-liquid phase-transfer alkylation reactions, we prepared the three set of catalysts based on phenanthrylamine (catalyst I), naphthylamine (catalyst-II) and benzylamine moiety (catalyst-III) as shown in scheme 1 and the catalytic studies were made under similar conditions.

Highest enantioselectivity was achieved when methyl iodide was used as the electrophile for the alkylation of tert-butyl glycinate benzophenone Schiff base using catalyst I (40\%, table 1, entry 4). Under similar conditions and using the same set of catalyst and the Schiff base, lower enantioselectivity $(\sim 15 \%)$ was noticed with benzyl bromide as electrophile (table 1, entry 18). Lower enantioselectivities were also observed when the ethyl ester was used as the substrate for alkylation compared to the tert-butyl glycine esters as evident from the catalytic runs 2,$4 ; 6,7 ; 9,10$ and 12,13 (table 1). Though the difference in ee content is very small, the trend of increased bulkiness giving better selectivity is evident both from the viewpoint of two different gylcine esters and different chiral palladium complexes used in our studies.

\subsection{Asymmetric alkylation of benzophenone imine glycinate under phase transfer conditions using optically active palladium complexes and the chiral ionic liquid}

Use of different solvents or mixed solvents (table 1) did not improve much the rate of reaction or the ee of the product mixture. We therefore thought of using the chiral ionic liquid (CIL) in combination with the optically active orthopalladated complexes and study their combined effects on product composition. When we added $1 \mathrm{ml}(9 \mathrm{~mol} \%)$ of the chiral ionic liquid ( $N, N$-dimethylephedriniumbis-(trifluoromethanesulfon)imidate) to the same reaction solution that

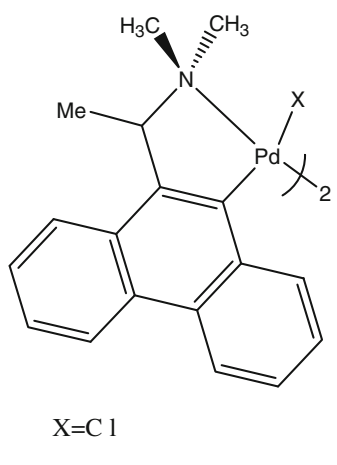

catalyst- I (S, enantiomer)

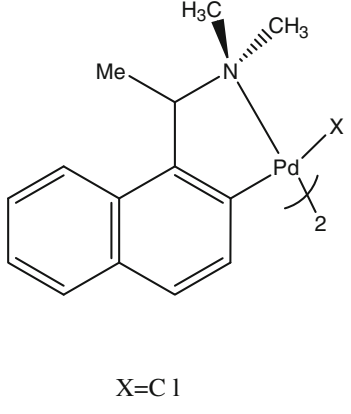

catalyst- II

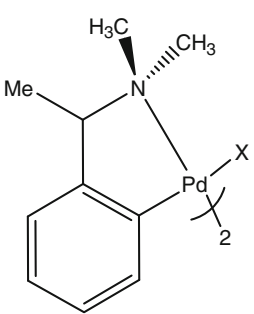

$\mathrm{X}=\mathrm{C} 1$

catalyst- III (S, enantiomer)

catalyst- IV (R, enantiomer)

Scheme 1. Orthopalladated complexes used as catalysts in enantioselective alkylation reaction. 
Table 1. Alkylation of glycine derivatives under phase transfer conditions using chiral orthopalladated complex.

\begin{tabular}{|c|c|c|c|c|c|c|}
\hline Entry No & Catalyst used & substrate & Electrophile & solvent & $*$ Pdt/Yield $(\%)^{\mathrm{a}}$ & ee $(\%)^{\mathrm{b}}$ \\
\hline 1 & Complex-I & $\mathrm{I}$ & $\mathrm{CH}_{3} \mathrm{I}$ & $\mathrm{CH}_{2} \mathrm{Cl}_{2}$ & $\mathrm{Ia} / 88$ & 30 \\
\hline 2 & Complex-I & I & $\mathrm{CH}_{3} \mathrm{I}$ & PhMe: $\mathrm{CHCl}_{3}$ & $\mathrm{Ia} / 86$ & 33 \\
\hline $3^{d}$ & Complex-I & I & $\mathrm{CH}_{3} \mathrm{I}$ & $\mathrm{PhMe}: \mathrm{CHCl}_{3}$ & $\mathrm{IIa} / 90$ & 33 \\
\hline 4 & Complex-I & II & $\mathrm{CH}_{3} \mathrm{I}$ & $\mathrm{CH}_{2} \mathrm{Cl}_{2}$ & IIa / 90 & 40 \\
\hline 5 & Complex-II & I & $\mathrm{CH}_{3} \mathrm{I}$ & $\mathrm{CH}_{2} \mathrm{Cl}_{2}$ & Ia / 88 & 26 \\
\hline 6 & Complex-II & I & $\mathrm{CH}_{3} \mathrm{I}$ & $\mathrm{PhMe}: \mathrm{CHCl}_{3}$ & Ia / 86 & 25 \\
\hline 7 & Complex-II & II & $\mathrm{CH}_{3} \mathrm{I}$ & PhMe: $\mathrm{CHCl}_{3}$ & $\mathrm{IIa} / 88$ & 30 \\
\hline 8 & Complex-III & II & $\mathrm{CH}_{3} \mathrm{I}$ & $\mathrm{CH}_{2} \mathrm{Cl}_{2}$ & IIa / 90 & 25 \\
\hline 9 & Complex-III & $\mathrm{I}$ & $\mathrm{CH}_{3} \mathrm{I}$ & $\mathrm{PhMe}: \mathrm{CHCl}_{3}$ & Ia / 85 & 18 \\
\hline 10 & Complex-III & II & $\mathrm{CH}_{3} \mathrm{I}$ & PhMe: $\mathrm{CHCl}_{3}$ & $\mathrm{IIa} / 85$ & 25 \\
\hline $11^{\mathrm{d}}$ & Complex-III & $\mathrm{I}$ & $\mathrm{CH}_{3} \mathrm{I}$ & $\mathrm{PhMe}: \mathrm{CHCl}_{3}$ & Ia / 88 & 20 \\
\hline 12 & Complex-IV & I & $\mathrm{CH}_{3} \mathrm{I}$ & $\mathrm{PhMe}: \mathrm{CHCl}_{3}$ & Ia / 84 & 22 \\
\hline 13 & Complex-IV & II & $\mathrm{CH}_{3} \mathrm{I}$ & $\mathrm{PhMe}: \mathrm{CHCl}_{3}$ & IIa / 86 & 28 \\
\hline 14 & Complex-IV & II & $\mathrm{BnBr}$ & $\mathrm{PhMe}: \mathrm{CHCl}_{3}$ & IIb / 78 & 12 \\
\hline 15 & Complex-III & II & $\mathrm{BnBr}$ & PhMe: $\mathrm{CHCl}_{3}$ & IIb / 74 & 10 \\
\hline 16 & Complex-II & II & $\mathrm{BnBr}$ & $\mathrm{PhMe}: \mathrm{CHCl}_{3}$ & IIb / 75 & 15 \\
\hline 17 & Complex-I & II & $\mathrm{BnBr}$ & $\mathrm{PhMe}: \mathrm{CHCl}_{3}$ & IIb / 78 & 17 \\
\hline 18 & Complex-I & II & $\mathrm{BnBr}$ & $\mathrm{CH}_{2} \mathrm{Cl}_{2}$ & IIb / 88 & 15 \\
\hline $19^{c}$ & Complex-I & II & $\mathrm{BnBr}$ & $\mathrm{CH}_{2} \mathrm{Cl}_{2}$ & $\mathrm{IIb} / 85$ & 12 \\
\hline $20^{\mathrm{d}}$ & Complex-I & II & $\mathrm{BnBr}$ & $\mathrm{CH}_{2} \mathrm{Cl}_{2}$ & $\mathrm{IIb} / 74$ & 17 \\
\hline
\end{tabular}

Alkylating agent: benzyl bromide/methyl iodide, $\mathrm{t}=20^{\circ} \mathrm{C}$, time $=12 \mathrm{~h}$

* Absolute configuration of products was determined to be $(S)$ from literature data for catalysts 1 -III and $(R)$ for catalyst IV

a: isolated crude oil before column chromatography

b: determined by ${ }^{1} \mathrm{H}-\mathrm{NMR}$, chiral HPLC and optical rotation studies

c: 5 eq of the electrophile used

d: a lower temperature of $-10^{\circ} \mathrm{C}$ was employed

yielded $30 \%$ enantiomeric excess (entry 1 , table 1 ) and stirred the reaction vigorously for 12 hours, we noticed a further increase in enantioselectivity. With chlorobridged phenanthrylamine orthopalladated complex as catalyst $(10 \mathrm{~mol} \%)$ and methyl iodide as the alkylating agent, ethyl-N-diphenylmethyleneglycinate (substrateI) yielded $(S)$-Ethyl-N-diphenyl methylene alaninate as the major isomer (table 2). The enantiomer excess improved to $42 \%$ when chiral ionic liquid (CIL) was used in addition to $\mathrm{CH}_{2} \mathrm{Cl}_{2}(1 \mathrm{~mL})$ and $50 \%$ aqueous $\mathrm{KOH}$ solution as base. Even $0.5 \mathrm{~mL}$ of the chiral ionic liquid produced the same result under similar conditions. With tert-butyl-N-diphenyl methyleneglycinate as substrate, the ee also increased to $48 \%$ keeping all other reagents and reaction conditions unchanged (entry 3, table 2). The same trend of ee increment was also noticed when benzyl bromide was used as the electrophile. With benzyl bromide as electrophile, tert-butyl-N-diphenylmethyleneglycinate (substrate-II) produced $(S)$-tert-butyl-N-diphenylmethylene-L-phenylalaninate as the major isomer (ee $28 \%$, entry 14 , table 2). Here too incorporation of the chiral ionic liquid improved the asymmetric induction by over $60 \%$ from the previous value ( $17 \%$, entry 17 , table 1$)$.
The enantiomeric R-complex of palladium (complexIV) was also studied as the catalyst in the enantioselective alkylation reaction of tert-butyl glycinate under similar optimised conditions to find the match-mismatch effect. There was the mismatch effect observed (R-enantiomer major in this case) although similar increment in ee $(\sim 60 \%)$ was noticed (entry 8 , table 2) when the chiral ionic liquid was added to the catalyst system. The ee was a mere $22 \%$ (entry 12, table 1) with no chiral ionic liquid added to the system. Even though the chiral ionic liquid alone cannot induce enantioselectivity under biphasic conditions (entry 19, table 2) it acts as a chiral booster to increase the ee when used along with the orthopalladated complexes. Even with a weaker base like $\mathrm{K}_{2} \mathrm{CO}_{3}$ and addition of 18-crown-6 to encapsulate the potassium ion, no asymmetric induction could be achieved with the ionic liquid alone. The charge distribution over the nitrogen atom in the chiral ionic liquid (preparation and structure provided as electronic Supplementary information) is so diffused that it fails to replace the potassium ion bound to the enolate and form an ion pair with the enolate ion. This is the first instance as far as literature survey says where a chiral ionic liquid acts as a chiral improver in 
Table 2. Alkylation of glycine derivatives under phase transfer conditions using chiral orthopalladated complex and chiral ionic liquid(CIL) at RT.

\begin{tabular}{|c|c|c|c|c|c|c|}
\hline Entry No & Catalyst used & substrate & electrophile & Solvent ${ }^{\mathrm{c}}$ & Yield $(\%)^{\mathrm{a}}$ & ee $(\%)^{\mathrm{b}}$ \\
\hline 1 & Complex-I & I & $\mathrm{CH}_{3} \mathrm{I}$ & $\mathrm{CH}_{2} \mathrm{Cl}_{2}$ & 88 & 30 \\
\hline 2 & Complex-I & I & $\mathrm{CH}_{3} \mathrm{I}$ & CIL & 88 & 42 \\
\hline 3 & Complex-I & II & $\mathrm{CH}_{3} \mathrm{I}$ & CIL & 92 & 48 \\
\hline 4 & Complex-I & I & $\mathrm{CH}_{3} \mathrm{I}$ & {$[\mathrm{Bmim}] \mathrm{PF}_{6}$} & 86 & 24 \\
\hline 5 & Complex-I & I & $\mathrm{CH}_{3} \mathrm{I}$ & {$[\mathrm{Bmim}] \mathrm{BF}_{4}$} & 88 & 26 \\
\hline 6 & Complex-II & I & $\mathrm{CH}_{3} \mathrm{I}$ & CIL & 88 & 35 \\
\hline 7 & Complex-III & I & $\mathrm{CH}_{3} \mathrm{I}$ & CIL & 92 & 30 \\
\hline 8 & Complex-IV & $\mathrm{I}$ & $\mathrm{CH}_{3} \mathrm{I}$ & CIL & 84 & 34 \\
\hline 9 & Complex-III & II & $\mathrm{CH}_{3} \mathrm{I}$ & {$[\mathrm{Bmim}] \mathrm{PF}_{6}$} & 85 & 24 \\
\hline 10 & Complex-III & II & $\mathrm{CH}_{3} \mathrm{I}$ & CIL & 88 & 35 \\
\hline 11 & Complex III & II & $\mathrm{CH}_{3} \mathrm{I}$ & {$[\mathrm{Emim}]\left[\mathrm{Tf}_{2} \mathrm{~N}\right]$} & 82 & 37 \\
\hline 12 & Complex-IV & II & $\mathrm{CH}_{3} \mathrm{I}$ & CIL & 85 & 35 \\
\hline 13 & Complex-IV & II & $\mathrm{CH}_{3} \mathrm{I}$ & {$[\mathrm{Emim}]\left[\mathrm{Tf}_{2} \mathrm{~N}\right]$} & 88 & 37 \\
\hline 14 & Complex-I & II & $\mathrm{C}_{6} \mathrm{H}_{5} \mathrm{CH}_{2} \mathrm{Br}$ & CIL & 90 & 28 \\
\hline 15 & Complex II & II & $\mathrm{C}_{6} \mathrm{H}_{5} \mathrm{CH}_{2} \mathrm{Br}$ & CIL & 88 & 25 \\
\hline 16 & Complex-III & II & $\mathrm{C}_{6} \mathrm{H}_{5} \mathrm{CH}_{2} \mathrm{Br}$ & CIL & 88 & 18 \\
\hline 17 & Complex-III & II & $\mathrm{C}_{6} \mathrm{H}_{5} \mathrm{CH}_{2} \mathrm{Br}$ & {$[\mathrm{Emim}]\left[\mathrm{Tf}_{2} \mathrm{~N}\right]$} & 85 & 20 \\
\hline 18 & Complex-IV & II & $\mathrm{C}_{6} \mathrm{H}_{5} \mathrm{CH}_{2} \mathrm{Br}$ & CIL & 85 & 20 \\
\hline 19 & - & II & $\mathrm{C}_{6} \mathrm{H}_{5} \mathrm{CH}_{2} \mathrm{Br}$ & CIL & 70 & Racemic \\
\hline 20 & - & II & $\mathrm{C}_{6} \mathrm{H}_{5} \mathrm{CH}_{2} \mathrm{Br}$ & $\mathrm{CIL}^{\mathrm{d}}$ & 74 & Racemic \\
\hline
\end{tabular}

a: isolated yield after column chromatography on basic alumina

b: determined by ${ }^{1} \mathrm{H}$ NMR and chiral HPLC analysis

${ }^{c}$ : ionic liquids were always used in association with dichloromethane and $50 \%$ aq $\mathrm{KOH}$ solution

d: 18 -crown-6 (10\%) was used along with $10 \mathrm{~mol} \%$ of CIL

asymmetric alkylation reactions. By changing the solvent composition with chiral ionic liquid and even by incorporation of room temperature ionic liquids like [Bmim] $] \mathrm{PF}_{6}$ (1-butyl-3-methyl imidazolium hexafluorophosphate) and [Bmim] $\mathrm{BF}_{4}$ (1-butyl-3-methyl imidazolium tetrafluoroborate), there was however no improvement on the product purity (entry 4 and 5 , table 2 ). The slight decrease in these cases may be attributed to the high viscous nature of the ionic liquids affecting the diffusion phenomenon in phase-transfer alkylation reactions.

We almost invoked the idea that it is the chirality of the chiral ionic liquid employed that led to enhancement of asymmetric induction until before we thought of using an ionic liquid having the same counterion as the $N, N$-dimethylephedrinium salt. Fortunately, we could use the $[\mathrm{Emim}]\left[\mathrm{Tf}_{2} \mathrm{~N}\right]$ ionic liquid (SolventInnovation, Germany, $99 \%$ pure) as a substitute to the chiral ionic liquid. Contrary to what we observed in case of $[\mathrm{Bmim}] \mathrm{PF}_{6}$, we found almost similar increment in ee as noticed in case of the chiral ionic liquid (entries 10,11 and 16,17, table 2). The thought that chirality of the chiral ionic liquid would be the factor for the rise in ee content soon subsided. We focused our work on more studies with different ionic liquids and simultaneous computer calculations to understand the nature of interactions and influence of counter-ion involved in such phase-transfer alkylation reactions.

Dramatic counter anion effects were observed in phase transfer catalysis in Michael addition system by Shibasaki et al. ${ }^{41}$ making it possible to further improve reactivity and selectivity. Since the (+) variety of the ephedrinium chiral ionic liquid could not be prepared employing the same procedure nor the desired ionic liquids with ephedrinium could be prepared by changing the anionic part, we examined the asymmetric alkylation reaction under phase-transfer conditions using the following ionic liquids as shown in table 3. Some of these ionic liquids were prepared and characterized by known literature methods and others were obtained from commercial sources. The conversion yield and the ee percentage change with and without the ionic liquids are presented here. For the sake of comparison we have used the same catalyst, same alkylating agent and same substrate under similar experimental conditions.

We tried to investigate what physicochemical property if not chirality of the ionic liquid could lead to almost 50\% increment in asymmetric induction. Parameters like density, viscosity, conductivity and solubility of the ionic liquids were therefore considered that could 
Table 3. Anionic effect of imidazolium based ionic liquids in catalytic asymmetric PT alkylation ${ }^{\mathrm{a}}$ promoted by palladium catalyst. $^{\text {b }}$

\begin{tabular}{|c|c|c|c|c|c|}
\hline Entry & Ionic liquid used ( $5 \mathrm{~mol} \%$ ) & Time (h) & Yield $(\%)^{\mathrm{c}}$ & $\operatorname{Ee}(\%)$ & Ee change $^{d}$ \\
\hline 1 & {$[\mathrm{BMIm}] \mathrm{Cl}$} & 12 & 84 & 28 & $+12 \%$ \\
\hline 2 & {$[\mathrm{BMIm}] \mathrm{BF}_{4}$} & 12 & 78 & 22 & $-12 \%$ \\
\hline 3 & {$[\mathrm{BMIm}] \mathrm{PF}_{6}$} & 12 & 74 & 20 & $-20 \%$ \\
\hline 4 & {$[\mathrm{BMIm}] \mathrm{PF}_{6}$} & 06 & 40 & 12 & $-52 \%$ \\
\hline 5 & {$[\mathrm{BMIm}] \mathrm{PF}_{6}$} & 24 & 78 & 22 & $-12 \%$ \\
\hline 6 & {$[\mathrm{BMIm}] \mathrm{MeHPO}_{3}$} & 12 & 80 & 24 & $-4 \%$ \\
\hline 7 & {$[\mathrm{BMIm}] \mathrm{MeSO}_{4}$} & 12 & 86 & 28 & $+12 \%$ \\
\hline 8 & {$[\mathrm{BMIm}] \mathrm{CH}_{3} \mathrm{COO}$} & 12 & 88 & 30 & $+20 \%$ \\
\hline 9 & {$[\mathrm{BMIm}] \mathrm{CH}_{3} \mathrm{COO}$} & 24 & 90 & 30 & $+20 \%$ \\
\hline 10 & {$[\mathrm{BMIm}] \mathrm{Tf}_{2} \mathrm{~N}$} & 12 & 88 & 36 & $+44 \%$ \\
\hline
\end{tabular}

\footnotetext{
${ }^{\mathrm{a}}$ : alkylating agent: $\mathrm{CH}_{3} \mathrm{I}$; substrate: tert-butylglycine Schiff base ester; $\mathrm{T}=20^{\circ} \mathrm{C}$; base: $50 \%$ aq $\mathrm{KOH}$

b: catalyst $=10 \mathrm{~mol} \%$ orthopalladated benzylamine complex(S-enantiomer)

${ }^{c}$ : isolated yield before column chromatography over basic alumina

d: compared with the run involving catalyst only under similar PT conditions (entry 10, table 1)
}

throw some light in this regard. There appears to be no overall correlation between density, viscosity and conductivity of the ionic liquids studied as revealed in literatures. ${ }^{42,43}$ The usual density and viscosity order of ionic liquids and other physicochemical parameters are given separately as supplementary information.

Although $\left(\mathrm{CF}_{3} \mathrm{SO}_{2}\right)_{2} \mathrm{~N}^{-}$has the highest density because of its high molecular mass, its viscosity is quite low indicating very weak attraction with the imidazolium cation. The high viscosity of [BMIm] $\mathrm{PF}_{6}$ is attributed to strong $\mathrm{C}(2)-\mathrm{H}-\mathrm{F}$ (imidazolium cation-anion) interaction as molecular structure of the ionic liquid shows much shorter $\mathrm{C}-\mathrm{H}-\mathrm{F}$ distances $(2.3 \AA$ ) compared to the $\mathrm{H}-\mathrm{F}$ Van der Waals distance of $2.7 \AA .{ }^{42}$ The weak interaction of $\mathrm{Tf}_{2} \mathrm{~N}^{-}$(bistrifluoromethanesulphonimidate anion) with the imidazolium cation will lead to greater Van der Waals attraction with the palladium complex in the organic layer. The steric congestion around the enolate ion would therefore lead to enhanced enantioselectivity. Though we expected the acetate and the chloride anions to interact with the palladium complex better because of their more localized charges, their increased solubility in the aqueous phase seems to nullify this advantage. Regarding solubility of ionic liquids in water at $20^{\circ} \mathrm{C}$, for the same $[\mathrm{BMIm}]^{+}$cation, $\mathrm{BF}_{4}^{-}, \mathrm{CF}_{3} \mathrm{SO}_{3}^{-}, \mathrm{CH}_{3} \mathrm{CO}_{2}^{-}, \mathrm{NO}_{3}^{-}$ and $\mathrm{Cl}^{-}$all display complete solubilities with water. $[\mathrm{BMIm}] \mathrm{PF}_{6}$ and $[\mathrm{BMIm}] \mathrm{Tf}_{2} \mathrm{~N}$, on the other hand, show very low solubility with water at room temperature. Since the solubility of $[\mathrm{BMIm}] \mathrm{Tf}_{2} \mathrm{~N}$ in water is quite low (1.4 mass\%), ${ }^{37}$ it will form a rich organic phase layer with $\mathrm{CH}_{2} \mathrm{Cl}_{2}$ in which the palladium catalyst is eventually dissolved. The anion would therefore have a greater chance to affect the stereo-geometry around the enolate ion formed at the interface of the aqueous and the organic layer. The electrophile would preferably approach the enolate ion-bound palladium complex from the site which is sterically less hindered. Anions like $\mathrm{BF}_{4}^{-}, \mathrm{CH}_{3} \mathrm{COO}^{-}, \mathrm{MeSO}_{4}^{-}$and $\mathrm{Cl}^{-}$because of their high solubility in the aqueous phase will have minimal effect in asymmetric induction process as witnessed by low ee increment (4-12\%, table 3) using these anions under phase-transfer alkylation conditions. Low viscosity of the ionic liquid and low solubility in the aqueous phase seems to be the right combination that works in favour of $[\mathrm{BMIm}] \mathrm{Tf}_{2} \mathrm{~N}$ in influencing enantioselectivity under present circumstances.

Computer studies have been made to examine the complexes formed and the nature of interactions between the chiral phase transfer catalyst and enolates that are known to be alkylated enantioselectively. A similar molecular recognition technique and computational tool have been employed by Lipkowitz et al. ${ }^{44}$ although their catalysts, used as asymmetric templates, were derived from the cinchona alkaloid family.

In the mechanistic model (scheme 2), contact ion pairing takes place between the anionic oxygen of the enolate and just one of the tetrahedral faces of the cationic nitrogen of the alkaloid species. In case of the chiral palladium complexes, the bridged dimer species spontaneously dissociates into the monomer solvent adduct and immediately combines with the Z-enolate to form a stable square planar species. This mode of bonding actually prevents the enolate component to be solvated or racemized in strongly basic media. The enolate with the catalyst is successfully transferred to the 


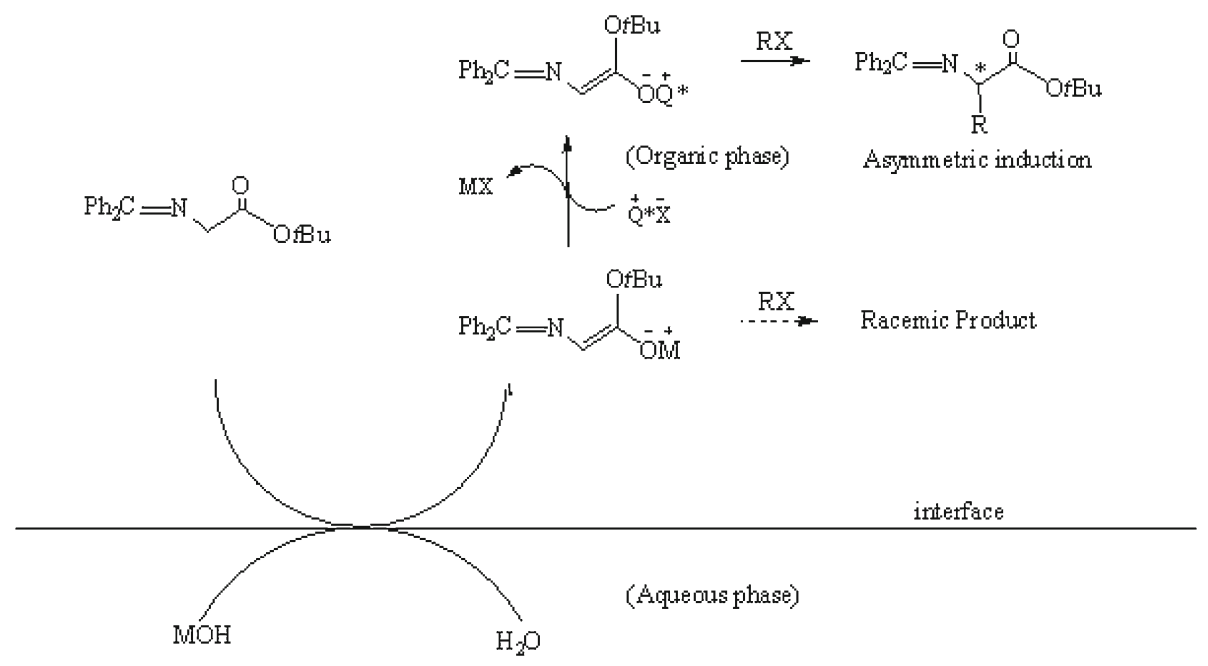

Scheme 2. General phase-transfer mechanism for asymmetric alkylation of active methylene compounds.

organic layer where it is lined up in the sterically most favourable geometry with the alkylating agent. Only the appropriate $r e$ face or the si face of the $\alpha$-carbon of the enolate is available for electrophilic attack leading to the observed $\mathrm{R}$ and $\mathrm{S}$ products respectively. The nitrogen atom of the chiral ionic liquid fails to dislodge the potassium or sodium ion bound to the enolate oxygen (scheme 2) and therefore only racemic products have been identified when $N, N$-dimethylephedriniumbis(trifluoromethanesulfon)imidate has been employed in the reaction medium without the palladium complexes as catalyst.

\subsection{Computational studies and analysis based on findings}

The interactions between the Z-enolate form of glycine derivative and palladium chiral catalysts were theoretically investigated using a Gaussian 03 program. ${ }^{45}$ tert-butyl-N-diphenylmethyleneglycinate has been taken as the reference substrate in all model computational studies with benzylamine orthopalladated complex as the catalyst. The electrophile is not accounted for in the modelling study and it is assumed that the low energy structures derived from the model are the ones leading to product.

The palladium catalyst with S-ligand (complex III) coordinates to Z-enolate in a square planar fashion. The complex with si face exposed to give S-enantiomer is calculated to be more stable than one with re face exposed to give R-enantiomer by $3.5 \mathrm{~kJ} / \mathrm{mol}$. Palladium catalyst with R-ligand (complex IV) also coordinates to Z-enolate in a square planar fashion. In this case, the complex with $r e$ face exposed to give R-enantiomer (figure 1b) is calculated to be more stable than one with $s i$ face exposed to give S-enantiomer (figure 1a) by almost $3.5 \mathrm{~kJ} / \mathrm{mol}$.

With the incorporation of the ionic liquids having trifluoromethane suphonyl imide anions to complex 3 favouring the S-enantiomer, coulombic and Van der Waals attraction of the palladium complex and the ionic

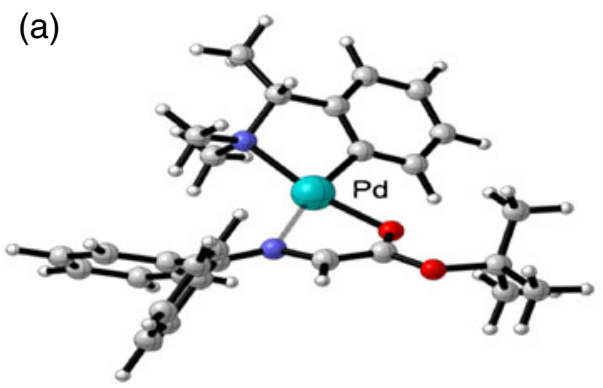

(b)

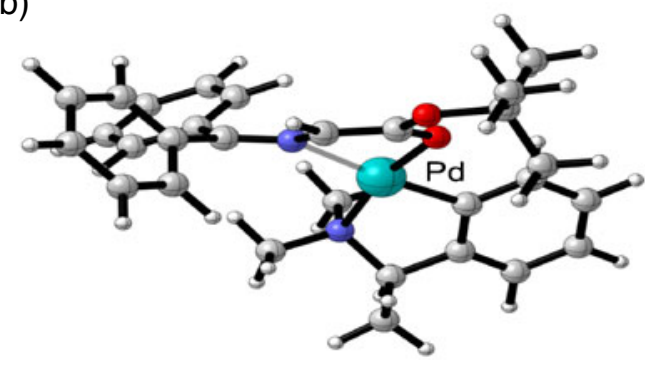

Figure 1. Chiral palladium benzyl complex coordinated to Z-enolate. (a) S-enantiomer; (b) R-enantiomer. 

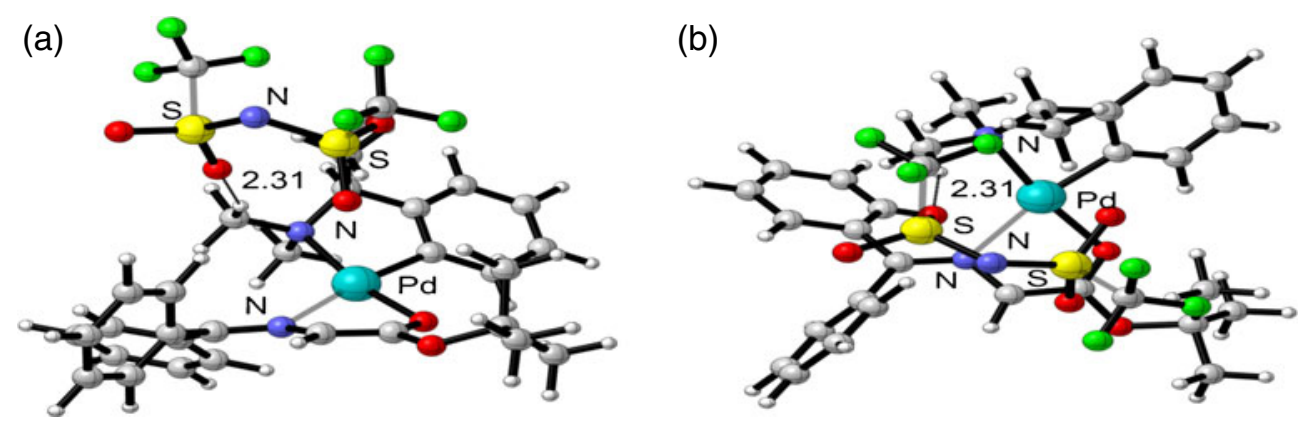

Figure 2. (a) Side view with anion coordination. (b) Top view with anion coordination.

liquid lead to a stereogenic center that favours the increment in ee (figure 2). The oxygen atoms bound to sulphur in the sulphonyl imide anion has been found in the model to interact with the $\mathrm{CH}_{3}$ groups of the palladium complex. ${ }^{1} \mathrm{H}$ NMR studies of the recovered ionic liquid phase after product extraction however did not reveal any changes in peak positions and so no coordination could be established through spectral studies. Both the side view (figure 2a) and the top view (figure $2 b$ ) of the coordinated anion of the ionic liquid with the palladium bound enolate however shows considerable steric congestion on the re face. Therefore forces the electrophile to approach from the much exposed $s i$ face leading to increased percentage of the S-enantiomer. $\mathrm{BF}_{4}^{-}$and $\mathrm{PF}_{6}^{-}$are not good coordinating anions ${ }^{42}$ that can stabilize a geometry for increased asymmetric induction and therefore no ee increase is noted on employing either $[\mathrm{Bmim}] \mathrm{BF}_{4}$ or $[\mathrm{Bmim}] \mathrm{PF}_{6}$ as chiral improvers.

\section{Conclusion}

A new design for asymmetric phase-transfer catalysts has been discussed. Orthopalladated chiral complexes in conjunction with ionic liquids were found to be effective catalysts in asymmetric phase transfer alkylation reactions. The rigid orthometallated phenanthrylamine moiety provided maximum asymmetric induction followed by the less constraint naphthylamine and benzylamine palladium complexes. Ionic liquids with trifluoromethansulphon imidate anion were found to improve the ee content in the range of $40-60 \%$. The use of metal catalysts in association with ionic liquids would throw new insights in enantioselective alkylation reactions. This combination holds much promise for asymmetric induction reactions under phase-transfer conditions and needs to be properly exploited with numerous choice of metal catalysts and ionic liquids available.

\section{Supplementary information}

Synthesis, ${ }^{1} \mathrm{H}$ NMR spectra and physicochemical properties of ionic liquids are provided as supplementary material (see www.ias.ac.in/chemsci).

\section{Acknowledgements}

Part of this project was supported by Science and Technology Department, Ministry of Korean Government and part by the University Grants Commission (UGC), Eastern Regional Office, India.

\section{References}

1. O'Donnell M J 1993 Asymmetric phase transfer reactions (ed) I Ojima, Catalytic asymmetric synthesis (New York: Verlag Chemie) chapter 8

2. Shioiri T 1997 Chiral phase transfer catalysis (eds) Y Sasson and R Neumann, Handbook of phase transfer catalysis (London: Blackie Academic \& Professional) chapter 14

3. O'Donnell M J, Delgado F, Hostettler C and Schwesinger R 1998 An efficient homogeneous catalytic enantioselective synthesis of $\alpha$-amino acid derivatives. Tetrahedron Lett. 398775

4. Chinchilla R, Mazon P and Najera C 2002 New dimeric anthracenyl-derived cinchona quaternary ammonium salts as phase-transfer catalysts for the asymmetric synthesis of $\alpha$-amino acids. Tetrahedron: Asymmetry 13 927

5. O'Donnell M J and Eckrich T M 1978 The synthesis of amino acid derivatives by catalytic phase-transfer alkylations. Tetrahedron Lett. 194625

6. O'Donnell M J, Bennett W D and Wu S 1989 The stereoselective synthesis of $\alpha$-amino acids by phase transfer catalysis. J. Am. Chem. Soc. 1112353

7. O'Donnell M J, Wu S and Huffman J C 1994 A new active catalyst species for enantioselective alkylation by phase-transfer catalysis. Tetrahedron $\mathbf{5 0} 4507$

8. Corey E J, Xu F and Noe M C 1997 A rational approach to catalytic enantioselective enolate alkylation using a structurally rigidified and defined chiral quaternary 
ammonium salt under phase transfer conditions. J. Am. Chem. Soc. 11912414

9. Corey E J, Noe M C and Xu F 1998 Highly enantioselective synthesis of cyclic and functionalized $\alpha$-amino acids by means of a chiral phase transfer catalyst. Tetrahedron Lett. 395347

10. Lygo B and Wainwright P G 1997 A new class of asymmetric phase-transfer catalysts derived from cinchona alkaloids-Application in the enantioselective synthesis of $\alpha$-amino acids. Tetrahedron Lett. 38 8595

11. Lygo B, Crosby J, Lowdon T R, Peterson J A and Wainwright P G 2001 Studies on the enantioselective synthesis of $\alpha$-amino acids via asymmetric phase transfer catalysis. Tetrahedron $\mathbf{5 7} 2403$

12. Elango S, Venugopal M, Suresh P S and Eni 2005 Contrast performance in catalytic activity-new cinchona phase transfer catalysts for asymmetric synthesis of $\alpha$-amino acids. Tetrahedron $\mathbf{6 1} 1443$

13. Chinchilla R, Najera C and Ortega F J 2007 Improved conditions for the asymmetric synthesis of amino acids using cinchona-derived anthracenylmethyl ammonium salts as chiral phase-transfer organocatalysts. Tetrahedron: Asymmetry 173423

14. Andrus M B, Ye Z and Zhang J 2005 Highly selective glycine phase-transfer catalysts using fluoroanthracenylmethyl cinchonidine catalysts. Tetrahedron Lett. 46 3839

15. Shirakawa S, Ueda M, Tanaka Y, Hashimoto $T$ and Maruoka K 2007 Design of binaphthyl-modified symmetrical chiral phase-transfer catalysts: substituent effect of 4,4',6,6'-positions of binaphthyl rings in the asymmetric alkylation of a glycine derivative. Chem. An Asian J. 21276

16. Siva A and Murugan E 2005 A new trimeric cinchona alkaloid as a chiral phase-transfer catalyst for the synthesis of asymmetric $\alpha$-amino acids. Synthesis 17 2927

17. Hashimoto T, Tanaka Y and Maruoka K 2003 Symmetrical 4,4',6,6' tetraalkylbinaphthyl substituted ammonium bromide as a new chiral phase-transfer catalyst. Tetrahedron: Asymmetry 141599

18. Kitamura M, Shirakawa S and Maruoka K 2005 Powerful chiral phase-transfer catalysts for the asymmetric synthesis of $\alpha$-alkyl and $\alpha \alpha$-dialkyl $\alpha$-amino acids. Angew Chem. Int. Ed. 44(10) 1549

19. Arakawa Y, Haraguchi N and Itsuno S 2008 An immobilization method of chiral quaternary ammonium salts onto polymer supports. Angew Chem. Int. Ed. 47 8232

20. O'Donnell M J and Wu S 1992 A catalytic enantioselective synthesis of $\alpha$-methyl aminoacid derivatives by phase-transfer catalysis. Tetrahedron: Asymmetry 3 591

21. Shi Q, Lee Y J, Kim M J, Park M K, Lee K, Song H, Chen M, Jeong B S, Park H G and Jew S S 2008 Highly efficient polymer supported phase transfer catalysts containing hydrogen bond inducing functional groups. Tetrahedron Lett. 491380

22. Wang X, Yin L, Yang T and Wang Y 2007 Synthesis of new dimeric-PEG-supported cinchona ammonium salts as chiral phase transfer catalysts for the alkylation of Schiff bases with water as solvent. Tetrahedron: Asymmetry $\mathbf{1 8} 108$

23. Ooi T and Maruoka K 2007 Recent advances in asymmetric phase-transfer catalysis-A review. Angew Chem. Int. Ed. 464222

24. Cozzi F 2006 Immobilization of organic catalysts: when, why and how. Adv. Synth. Catal. 3481367

25. Chinchilla R, Mazon P and Najera C 2004 Polystyrene anchored cinchona ammonium salts: easily recoverable phase-transfer catalysts for the asymmetric synthesis of $\alpha$-amino acids. Adv. Synth. Catal. 3461186

26. Belokon Y N, Davies R G and North M 2000 A practical asymmetric synthesis of $\alpha$-methyl $\alpha$-amino acids using a chiral $\mathrm{Cu}$-Salen complex as a phase transfer catalyst. Tetrahedron Lett. 417245

27. Mukherjee D K and Ghosh N 2008 Enantioselective phase-transfer alkylation using orthopalladated complex in chiral ionic liquid. Catal. Commun. 940

28. Kim D Y, Kim D W, Im J K, Kim H, Lee H, Cheong M, Kim H S and Mukherjee D K 2010 Orthopalladated complexes as phase-transfer catalysts for asymmetric alkylation of achiral Schiff base esters. Trans. Met. Chem. 35 249. (doi:10.1007/s11243-010-9416-4)

29. Wilkes J S 2002 A short history of ionic liquids from molten salts to neoteric solvents. Green Chem. 473

30. Sheldon R A 2001 Catalytic reactions in ionic liquids. Chem. Commun. 2399

31. Wasserscheid P and Keim W 2000 Ionic liquids-new solutions for transition metal catalysis. Angew Chem. Int. Ed. 393772

32. Wheeler C, West K N, Liotta C L and Eckert C A 2001 Ionic liquids as catalytic green solvents for nucleophilic displacement reactions. Chem. Commun. 887

33. Kowtoniuk W E, MacFarland D K and Grover G N 2005 Combining chiral elements: a novel approach to asymmetric phase-transfer catalyst design. Tetrahedron Lett. 465703

34. Baudequin C, Bregeon D, Levillain J, Guillen F, Plaquevent J C and Gaumont A C 2005 Chiral ionic liquids, a renewable for the chemistry of chiral solvents: design, synthesis and applications for chiral recognition and asymmetric synthesis. Tetrahedron: Asymmmetry 16 3921

35. Baudequin C, Baudoux J, Levillain J, Cahard D, Gaumont A C and Plaquevent J C 2003 Ionic liquids and chirality: opportunities and challenges. Tetrahedron: Asymmmetry $\mathbf{1 4} 3081$

36. Branco L C, Gois P M P, Lourenco N M J, Kurteva V B and Afonso C A M 2006 Simple transformation of crystalline chiral natural anions to liquid medium and their use to induce chirality. Chem. Commun. 2371

37. Bonhote P, Dias A P, Papageorgiou N, Kalyanasundaram K and Gratzel M 1996 Hydrophobic, highly conductive ambient-temperature molten salts. Inorg. Chem. 351168

38. Wilkes J S and Zaworotko M J 1992 Air and water stable 1-ethyl-3methyimidazolium based ionic liquids. $J$. Chem. Soc. Chem. Commun. 965

39. O'Donnell M J, Boniece J M and Earp S E 1978 The synthesis of amino acids by phase-transfer reactions. Tetrahedron Lett. 302641 
40. Wasserscheid P, Bosman A and Bolm C 2002 Synthesis and properties of ionic liquids derived from the chiral pool. Chem. Commun. 200

41. Ohshima T, Shibuguchi T, Fukuta Y and Shibasaki M 2004 Catalytic asymmetric phase-transfer reactions using tartrate-derived asymmetric two-center organocatalysts. Tetrahedron $\mathbf{6 0} 7743$

42. Fredlake C P, Crosthwaite J M, Hert D G, Aki S N V and Brennecke J F 2004 Thermophysical properties of imidazolium-based ionic liquids. J. Chem. Eng. Data 49 954
43. Holbrey J D, Visser A E and Rogers R D 2003 Solubility and solvation in ionic liquids (eds) $\mathrm{P}$ Wasserscheid and $\mathrm{T}$ Welton, Ionic liquids in synthesis (Wiley-VCH) chapter 3.3

44. Lipkowitz K B, Cavanaugh M W, Baker B and O'Donnell M J 1991 Theoretical studies in molecular recognition: asymmetric induction of benzophenone imine ester enolates by the benzylcinchonium ion. $J$. Org. Chem. 565181

45. Frisch M J, Trucks G W, Schlegel H B et al. 2003 Gaussian 03, revision c.02 (Pittsburgh, PA: Gaussian Inc.) 